\title{
Etude in situ par analyse EXAFS d'un catalyseur de reformage $\mathrm{Pt}-\mathrm{Sn} / \mathrm{Al}_{2} \mathrm{O}_{3}-\mathrm{Cl}$
}

\author{
P. Bourges, F. Garin, G. Maire, G. Szabo*, M. Laborde*, R. Loutaty* et D. Bazin** \\ LERCSI, Université Louis Pasteur, 4 rue Blaise Pascal, 67000 Strasbourg, France \\ * TOTAL, Centre de Recherches, BP. 27, 76700 Harfleur, France \\ ** LURE, Université Paris Sud, Bât. 209D, 91405 Orsay, France
}

\begin{abstract}
Résumé : La connaissance de l'environnement du platine pour des catalyseurs bimétalliques $\mathrm{Pt}-\mathrm{Sn} / \mathrm{Al}_{2} \mathrm{O}_{3}-$ Cl oxydés ou réduits, peut permettre d'expliquer les propriétés catalytiques de la phase métallique. Les caractéristiques de l'environnement du platine peuvent être fournies par une analyse EXAFS du catalyseur, même si la teneur massique en platine est inférieure à $0,5 \%$. Dans ce travail, nous avons étudié un catalyseur de reformage $\mathrm{Pt}$ - $\mathrm{Sn} / \mathrm{Al}_{2} \mathrm{O}_{3}-\mathrm{Cl}$ à l'état oxydé et réduit par analyse EXAFS in situ. Sur le catalyseur oxydé, nous avons mis en évidence la formation d'oxydes du type $\left[\mathrm{PtO}_{\mathrm{x}} \mathrm{Cl}_{\mathrm{y}}\right]$ et la proximité des oxydes de platine et d'étain. L'analyse EXAFS réalisée in situ sur le catalyseur réduit montre que $20 \%$ du platine forme un alliage du type PtSn avec l'étain.
\end{abstract}

\begin{abstract}
The knowledge of the platinum environment on bimetallic catalysts $\mathrm{Pt}-\mathrm{Sn} / \mathrm{Al}_{2} \mathrm{O}_{3}-\mathrm{Cl}$, when the catalyst is either oxidised or reduced, is necessary in order to explain the catalytical properties. EXAFS gives characteristics of platinum environment, even if the platinum content is lower than $0.5 \%$. In this work, we have carried out a study by in situ EXAFS of a reforming catalyst Pt-Sn/ $/ \mathrm{Al}_{2} \mathrm{O}_{3}-\mathrm{Cl}$. On one hand, on oxidised catalyst, we have evidenced the formation of $\left[\mathrm{PtO}_{\mathrm{x}} \mathrm{Cl}_{\mathrm{y}}\right]$ oxide and the proximity of platinum oxides and tin oxides. On the other hand, on reduced catalyst, in situ EXAFS has shown that $20 \%$ of platinum is engaged in a PtSn alloy.
\end{abstract}

\section{INTRODUCTION}

Les catalyseurs bimétalliques présentent un très grand intérêt en catalyse hétérogène et sont utilisés communément en reformage. Ces systèmes sont principalement constitués d'un métal actif le platine et d'un second métal pouvant être inactif comme l'étain.

Dans des conditions industrielles de reformage, le catalyseur $\mathrm{Pt}-\mathrm{Sn} / \mathrm{Al}_{2} \mathrm{O}_{3}-\mathrm{Cl}$ est périodiquement régénéré afin de brûler les résidus hydrocarbonés formés à la surface du catalyseur. Durant la régénération, le catalyseur est calciné ; les métaux sont donc oxydés au cours de la régénération. Le platine se trouve alors sous forme d'oxyde $\mathrm{PtO}_{2}$ et l'étain sous forme d'oxyde $\mathrm{SnO}_{2}$ [1]. Mais en présence de chlore, des oxydes du type $\left[\mathrm{PtIV}^{I V} \mathrm{O}_{\mathbf{x}} \mathrm{Cl}_{\mathbf{y}}\right.$ peuvent se former [2]. La nature des oxydes formés et la proximité de ces différents oxydes sont des paramètres qui influent très fortement sur la dispersion et la composition de la phase métallique formée après réduction du catalyseur [3][4].

Après la régénération, le catalyseur est réduit sous hydrogène avant de réaliser un nouveau cycle de travail. Les différentes études physiques (XPS, Mössbauer) réalisées sur des catalyseurs $\mathrm{Pt}-\mathrm{Sn} / \mathrm{Al}_{2} \mathrm{O}_{3}$ réduits montrent que l'étain est presque uniquement présent à l'état $\mathrm{Sn}^{\mathrm{II}}$, en forte interaction avec l'alumine [5][6][7]. L'étain est un promoteur de la 
dispersion du platine, ce dernier formant de petites cristallites liées à l'alumine par l'intermédiaire d'ions $\mathrm{Sn}^{\mathrm{II}}$ [8]. La formation d'étain à l'état métallique engagé dans un alliage avec le platine, semble dépendre de la méthode de préparation du catalyseur et des procédures de réduction [9][10][11]. De plus, les faibles teneurs en métaux ( $<0,5 \%$ en masse) rendent la détection d'étain à l'état métallique délicate par XPS [12] ou par spectroscopie Mössbauer [13]. Par contre l'analyse EXAFS peut permettre d'étudier des échantillons présentant des teneurs en métaux comprises entre 0,2 et $0,5 \%$ en masse[14]. L'EXAFS permet de connaître l'environnement du platine ou de l'étain en ayant accès aux informations concernant la nature, le nombre et les distances des premiers voisins [15][16].

L'objectif de ce travail est l'étude d'un catalyseur de reformage industriel : $\mathrm{Pt}$ - $\mathrm{Sn} / \mathrm{Al}_{2} \mathrm{O}_{3}$ Cl par spectroscopie d'absorption X, EXAFS. Ce catalyseur présente des teneurs massiques en platine et en étain inférieures à $0,5 \%$ et une teneur massique en chlore voisine de $1 \%$. Ce catalyseur va être étudié sous forme oxydée et sous forme réduite dans des conditions d'utilisation industrielle : sous flux d'hydrogène à $450^{\circ} \mathrm{C}$. Les analyses EXAFS réalisées au seuil du platine doivent permettre de connaître l'environnement du platine sur un catalyseur après régénération et sur un catalyseur au départ d'un cycle de travail.

\section{PROCÉDURE OPÉRATOIRE}

Les spectres EXAFS sont réalisés au L.U.R.E. à Orsay. Le rayonnement synchrotron du L.U.R.E. provient de l'anneau de stockage du DCI à $1,85 \mathrm{GeV}$ avec un courant moyen de 250 $\mathrm{mA}$. Les données de spectroscopie d'absorption X sont collectées sur la station "EXAFS-4".

L'étude de nos échantillons est réalisée au seuil LIII du platine (11564 ev) en utilisant un monochromateur pas à pas avec des cristaux de $\mathrm{Si}$ [111] et deux chambres d'ionisation comme détecteurs. Les spectres sont obtenus en transmission en balayant la zone d'énergie 11450 à 12100 ev par pas de $1 \mathrm{ev}$. Le réglage du monochromateur est réalisé avec une feuille de platine.

L'échantillon de catalyseur $\mathrm{Pt}-\mathrm{Sn} / \mathrm{Al}_{2} \mathrm{O}_{3}-\mathrm{Cl}$ à analyser est sous forme oxydée. Il est d'abord broyé puis placé dans un four construit par le LERCSI. Ce four est placé entre les deux chambres d'ionisation, il permet le passage du rayonnement sur l'échantillon (l'épaisseur de l'échantillon traversée par le rayonnement est de $5,8 \mathrm{~mm}$ ). Cette installation permet de réaliser un traitement in situ du catalyseur sous atmosphère et sous température contrôlées, pendant l'acquisition des spectres "EXAFS".

L'étude EXAFS du catalyseur $\mathrm{Pt}-\mathrm{Sn} / \mathrm{Al}_{2} \mathrm{O}_{3}-\mathrm{Cl}$ est réalisée selon cette procédure, à pression atmosphérique : - analyse "EXAFS" de l'échantillon oxydé à température ambiante,

- réduction de l'échantillon $2 \mathrm{~h}$ à $450^{\circ} \mathrm{C}$ sous hydrogène,

- analyse "EXAFS" de l'échantillon à $450^{\circ} \mathrm{C}$ sous hydrogène,

- baisse de la température de 450 à $400^{\circ} \mathrm{C}$ sous hydrogène,

- analyse "EXAFS" de l'échantillon à $400^{\circ} \mathrm{C}$ sous hydrogène,

Afin d'interpréter les spectres "EXAFS" de nos échantillons, les spectres "EXAFS" des composés références suivants ont été enregistrés à température ambiante et aussi à différentes températures jusqu'à $450^{\circ} \mathrm{C}$ : une feuille de platine métallique, l'oxyde $\beta-\mathrm{PtO}_{2}$, l'acide $\mathrm{H}_{2} \mathrm{PtCl}_{6}$ et l'alliage PtSn.

\section{TRAITEMENT DES SPECTRES EXAFS}

L'interprétation des spectres d'absorption $X$ réalisés au seuil du platine est axée sur la partie du spectre située entre 40 et 1000 ev après le seuil d'absorption; cette zone correspond aux oscillations EXAFS. Les spectres obtenus sont traités à partir de la chaîne de programmes mise au point pour Macintosh [17]. 


\subsection{Démarche suivie}

A partir des spectres "EXAFS" obtenus avec les différents échantillons, les transformées de Fourier sont construites. Ces transformées de Fourier vont être simulées en vue de connaître le nombre, les distances et la nature des voisins.

L'utilisation de la formule théorique de "l'EXAFS" pour la détermination des distances $\mathrm{R}_{\mathrm{j}}$ entre l'atome central et l'atome rétrodiffuseur $\mathrm{j}$, implique la connaissance des termes de déphasage atomique (phase et amplitude). Deux possibilités sont à envisager :

- utiliser les phases et amplitudes théoriques (Mac Kale, Lee et al.). Mais nos spectres ont été pris à différentes températures, et ces fichiers théoriques ne nous donnent pas d'informations sur le facteur de désordre, "Debye-Waller", à appliquer.

- mesurer la phase et l'amplitude d'un composé modèle de structure cristalline connue et transférer ce déphasage expérimental dans l'interprétation "EXAFS" des échantillons où le nombre et la distance des voisins n'est pas connue.

\subsection{Détermination des phases et amplitudes expérimentales}

\subsubsection{Composés de référence}

Les composés modèles utilisés sont ceux proches, du point de vue structure cristalline, des espèces supposées présentes dans les échantillons étudiés. Ces composés sont : une feuille de platine métallique, l'oxyde $\beta-\mathrm{PtO}_{2}$, l'acide $\mathrm{H}_{2} \mathrm{PtCl}_{6}$, l'alliage PtSn. L'alliage PtSn non supporté utilisé comme référence dans ce travail a été au préalable caractérisé par des analyses RX, "XPS" et "EXAFS" (au seuil du platine et de l'étain) ; la phase métallique servant de référence correspond bien à un alliage PtSn.

\subsubsection{Extraction des transformées de Fourier}

Les transformées de Fourier doivent être extraites du signal "EXAFS" en utilisant le même type de fenêtre et les mêmes bornes, sur tous les spectres analysés : échantillons de référence et échantillons analysés. Dans cette étude, toutes les transformées de Fourier ont été extraites du signal "EXAFS" dans ces conditions :

- type de fenêtre : HANNING

- bornes de la fenêtre : $\quad 3,3-3,9-9,3-10,5 \mathrm{~K}\left(\AA^{-1}\right)$

Les figures ci-dessous représentent les modules des transformées de Fourier (notées T.F.) des composés de référence au seuil LIII du platine. L'unité en ordonnée est arbitraire (UA).

\subsubsection{Module de la T.F. de $\beta-\mathrm{PtO}_{2}$}

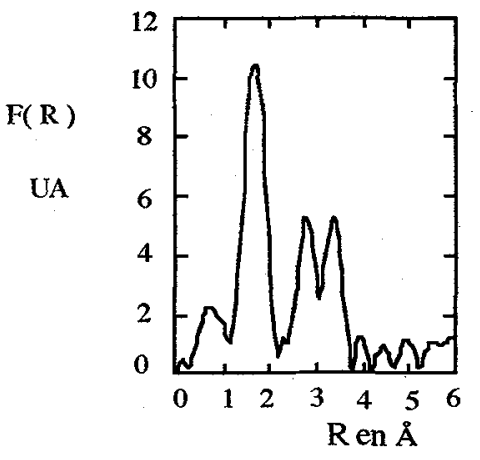

Figure 1: Module de la T.F. de $\beta-\mathrm{PtO}_{2}$ $\mathrm{F} T$ modulus for $\beta-\mathrm{PtO}_{2}$

Le pic principal de la T.F. de $\beta-\mathrm{PtO}_{2}$ est compris entre 1,24 et $2,15 \AA$, il est associé à 6 atomes d'oxygène à une distance de $2,04 \AA$ par rapport au platine. 


\subsubsection{Module de la T.F. de $\mathrm{H}_{2} \mathrm{PtCl}_{6}$}

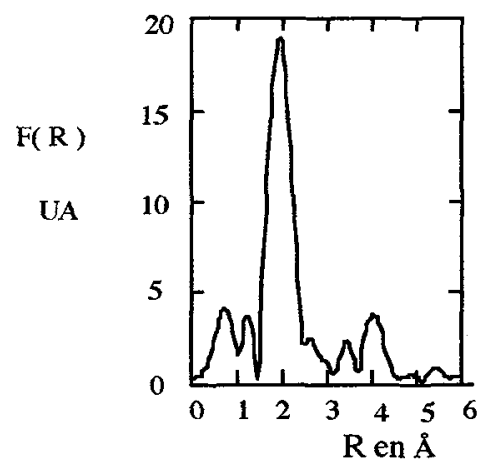

Figure 2: Module de la T.F. de $\mathrm{H}_{2} \mathrm{PtCl}_{6}$ F T modulus for $\mathrm{H}_{2} \mathrm{PtCl}_{6}$

Le pic principal de la T.F. de $\mathrm{H}_{2} \mathrm{PtCl}_{6}$ est compris entre 1,5 et $2,43 \AA$, il correspond à 6 atomes de chlore en proches voisins par rapport au platine, à une distance de $2,32 \AA ̊$.

\subsubsection{Module de la T.F. du platine métallique}

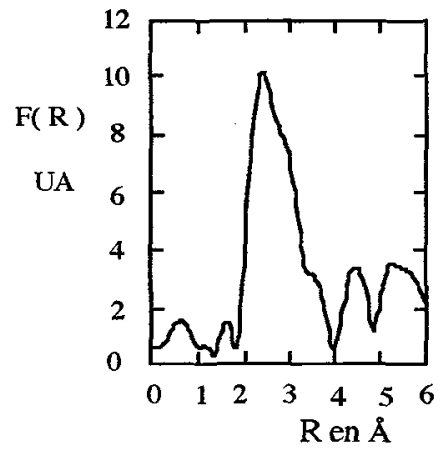

Figure 3 : Module de la T.F. du platine métallique F T modulus for metallic platinum

Sur le module de la transformée de Fourier du platine, le pic principal se situe entre 1,50 et $3,14 \AA$. Il est caractéristique de 12 atomes de platine en proches voisins, distants de $2,77 \AA$ par rapport au platine.

\subsubsection{Module de la T.F. de l'alliage PtSn}

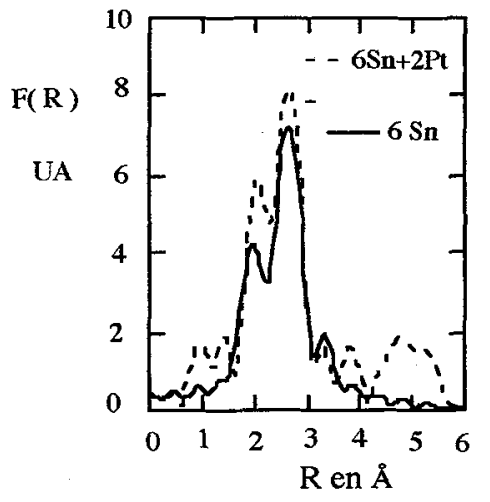

Figure 4 : Module de la T.F. de l'alliage PtSn F T modulus for the PtSn alloy

Le madule de la transformée de Fourier de PtSn est représenté en pointillés.

Le pic principal du module de la transformée de Fourier, obtenu à partir du spectre "EXAFS" de l'alliage PtSn, contient à la fois la contribution de deux atomes de platine à une distance de $2,72 \AA$ et celle de six atomes d'étain à une distance de $2,74 \AA$ (courbe en pointillés). Ces deux contributions sont difficiles à séparer.

Pour accéder uniquement à la contribution de l'étain, nous avons soustrait au spectre la contribution de deux atomes de platine. La contribution de deux atomes de platine situés à $2,72 \AA$ de l'atome central a été simulée en utilisant les phases et amplitudes expérimentales du platine (obtenues à partir du spectre "EXAFS" réalisé sur la feuille de platine métallique).

Par soustraction de la contribution des deux atomes de platine, on obtient ainsi le spectre correspondant à 6 atomes d'étain situés à $2,74 \AA$ de l'atome de platine central. Sur le graphe, la courbe en trait continu représente le module de la transformée de Fourier de six atomes d'étain situés à 2,74 Å d'un atome de platine. 


\subsubsection{Extraction de la phase et de l'amplitude}

Nous disposons des spectres "EXAFS" réalisés sur les références citées ci-dessus à diverses températures ( $\mathrm{T}$ ambiante $\rightarrow 420^{\circ} \mathrm{C}$ ). Pour chaque référence, les transformées de Fourier obtenues aux diverses températures ont été superposées afin de déterminer avec plus de précision les bornes du pic principal.

Ensuite, à partir de la transformée de Fourier du composé de référence obtenue à température ambiante, on réalise une "Fourier inverse" en ne sélectionnant que la zone du pic principal. Le signal "EXAFS" ainsi obtenu correspond alors aux $n_{j}$ premiers voisins $\mathrm{j}$ situés à une distance $R_{j}$. Les fichiers experimentaux phase et amplitude de l'atome $j$ sont alors extraits de ce spectre "EXAFS".

On obtient ainsi les fonctions phases et amplitudes expérimentales obtenues au seuil LIII du platine à température ambiante, pour les voisins suivants : $\mathrm{Pt}-\mathrm{Pt}, \mathrm{Pt}-\mathrm{O}, \mathrm{Pt}-\mathrm{Cl}, \mathrm{Pt}-\mathrm{Sn}$.

L'alliage utilisé pour extraire les phases et amplitudes entre le platine et l'étain est l'alliage $\mathrm{PtSn}$. Mais sur nos échantillons, il peut se former un autre alliage : $\mathrm{Pt}_{3} \mathrm{Sn}$. Les premiers voisins du platine ont alors 8 voisins $P t$ à $2,82 \AA$ et 4 voisins $S n$ à $2,82 \AA$ [18].

Il a été vérifié que les fichiers phases et amplitudes de Pt-Sn obtenus à partir de la référence expérimentale PtSn permettent de simuler le pic principal du module de la transformée de Fourier du spectre "EXAFS" réalisé sur l'alliage Pt 3 Sn [18].

\subsection{Effet de la température}

Comme le phénomène "EXAFS" est sensible au désordre thermique et statique, les effets de désordre qui sont pris en compte par le facteur de Debye-Waller doivent être intégrés à l'analyse "EXAFS". Les échantillons références obtenus à diverses températures vont permettre de déterminer pour chaque composé l'évolution du Debye-Waller en fonction de la température. Les spectres obtenus à haute temperature sont simulés avec les fichiers phases et amplitudes du composé correspondant, obtenus à température ambiante. On peut ainsi construire les courbes représentant l'évolution du facteur Debye-Waller en fonction de la température.

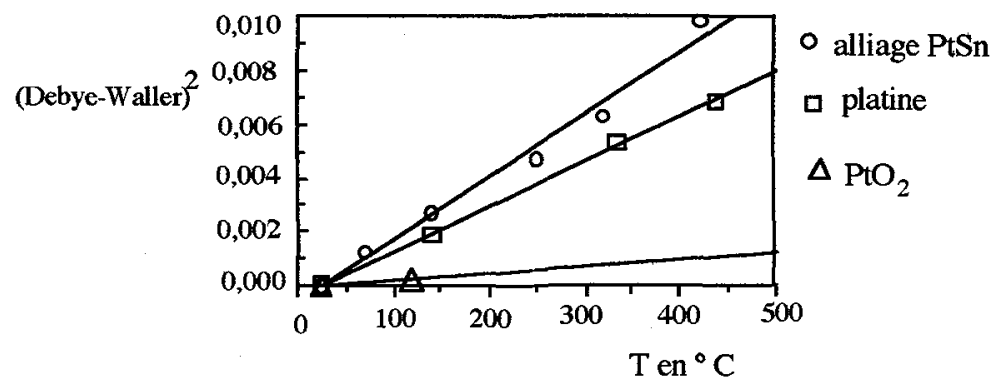

Figure 5: $\quad$ Évolution du facteur Debye-Waller en fonction de la température Variation of the Debye-Waller factor versus the temperature

On observe que l'évolution du facteur Debye-Waller en fonction de la température dépend du type de composé. 


\subsection{Simulation de la zone des premiers voisins du platine}

La simulation est réalisée sur la Fourier inverse de la zone des premiers voisins du platine. Sur tous les échantillons oxydés et réduits, la zone de la transformée de Fourier simulée est comprise entre 1,2 et $3,4 \AA$ A. Elle correspond à la zone des premiers voisins $\mathrm{O}, \mathrm{Cl}, \mathrm{Pt}$ et $\mathrm{Sn}$. $\mathrm{La}$ simulation peut être réalisée sur une partie seulement du pic global des premiers voisins en vue d'observer plus spécialement les voisins oxygène et chlore ou les voisins platine et étain. Mais la précision des résultats fournie par cette simulation partielle dépend de la résolution de ces deux pics. Cette simulation partielle donne un ordre d'idée sur le nombre de voisins et permet de constater l'absence ou la présence d'un des éléments dans le voisinage du platine. Dans certains cas, seule la simulation de la zone globale des premiers voisins est possible.

Les résultats $n_{j}$ et $R_{j}$ proposés pour chaque voisin $j$ du platine sont donnés avec comme précision :

$$
\begin{aligned}
& -\Delta n_{\mathbf{j}}=0,15 \cdot n_{j} \\
& -\Delta R_{j}=0,02 \AA
\end{aligned}
$$

\section{RÉSULTATS}

\subsection{Résultats obtenus sur l'échantillon oxydé}

Les spectres "EXAFS" de l'échantillon oxydé ont été enregistrés à $25^{\circ} \mathrm{C}$. Lors de la simulation de ces spectres, le facteur de Debye-Waller est toujours nul.

Le module de la transformée de Fourier obtenu sur le catalyseur neuf oxydé est représenté cidessous. La simulation est réalisée sur les pics représentant les premiers voisins : 1,2 à $3,2 \AA$.

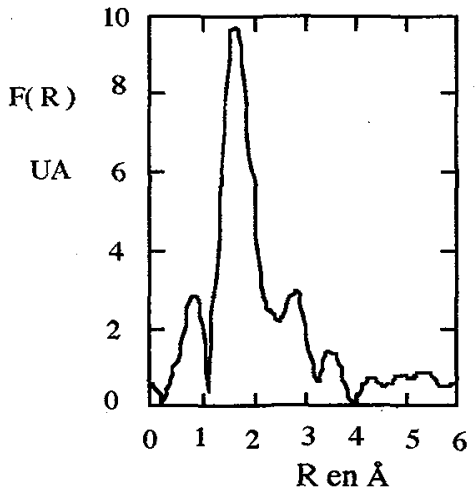

Figure 6 : Module de la TF du catalyseur oxydé

F T modulus for the oxidized catalyst

Cette zone peut être décomposée en deux :

$-1,2-2,2 \AA$ : voisins $\mathrm{Cl}$ ou $\mathrm{O}$

$-2,5-3,2 \AA$ : voisins Pt ou $\mathrm{Sn}$

La simulation de la zone $2,5-3,2 \AA$ n'est pas possible sur cette transformée de Fourier, car ce pic n'est pas suffisamment séparé du pic de l'oxygène.

Simulation de la Fourier inverse de la partie : $1,2-2,2 \AA$ :

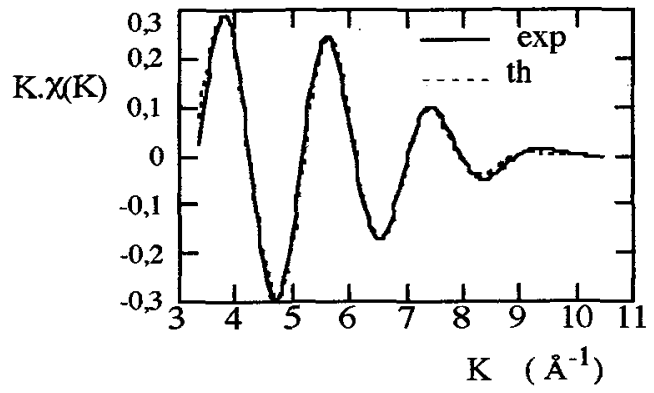

Figure 7: $\quad$ Catalyseur oxyde Fourier inverse ; $1,2-2,2 \AA$

Oxidized catalyst

Inverse $F T: 1.2-2.2 \AA$

Résultats :

$\begin{array}{ll}5,6 \text { oxygène } & \text { à } 2,01 \AA \\ 1,7 \text { chlore } & \text { à } 2,31 \AA\end{array}$


Simulation de la Fourier inverse de la zone des premiers voisins : 1,2 - 3,2 $\AA$ :

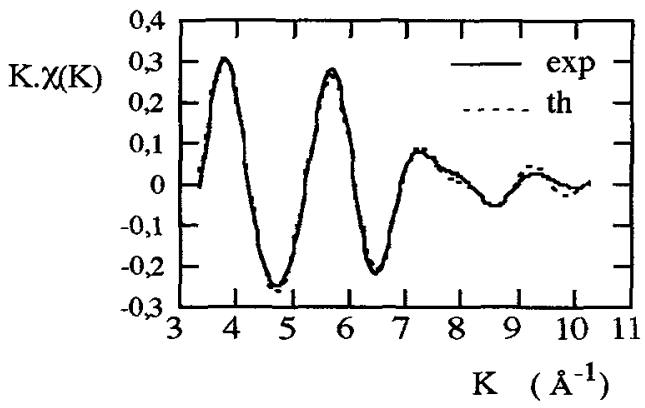

Figure 8: $\quad$ Catalyseur oxydé

Fourier inverse ; $1,2-3,2 \AA$

Oxidized catalyst

Inverse F T : $1.2-3.2 \AA$

Résultats :

4,9 oxygène

à $2,01 \AA$

2,3 chlore

à $2,31 \AA$

2,4 platine

à $2,77 \AA$

2,0 étain

à $2,82 \AA$

\subsection{Résultats obtenus sur l'échantillon réduit}

Des spectres "EXAFS" ont été réalisés après la réduction à 450 et $400^{\circ} \mathrm{C}$.

Lors de la simulation des spectres obtenus, le facteur de Debye-Waller est nul lorsque la température d'analyse est de $25^{\circ} \mathrm{C}$, mais il doit être ajusté pour chacun des éléments présents autour du platine lorsque la température d'analyse est de 400 ou $450^{\circ} \mathrm{C}$.

Les simulations réalisées sur les spectres obtenus à 450 et $400^{\circ} \mathrm{C}$ sont affinées de manière à vérifier les points suivants : la simulation des spectres obtenus à 400 et $450^{\circ} \mathrm{C}$ doit être réalisée en gardant le même nombre de voisins, aux mêmes distances, en diminuant seulement les valeurs des Debye-Waller (noté DB) lorsque la température passe de 450 à $400^{\circ} \mathrm{C}$

Le module de la transformée de Fourier obtenu sur le catalyseur neuf réduit est représenté cidessous. La simulation est réalisée sur les pics représentant les premiers voisins : 1,2 à 3,2 $\AA$.

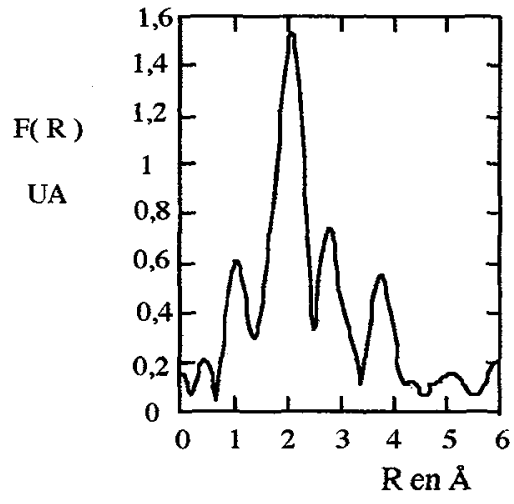

Figure 9: Module de la TF du catalyseur réduit F T modulus for the reduced catalyst

Cette zone peut être décomposée en deux :

$-1,3-2,3 \AA$ : voisins $\mathrm{Cl}$ ou $\mathrm{O}$

$-2,3-3,4 \AA$ : voisins $\mathrm{Pt}$ ou $\mathrm{Sn}$

La simulation de la zone $2,3-3,4 \AA$ n'est pas réalisable parce que le pic métallique n'est pas suffisamment séparé du pic de l'oxyde. 
Simulation de la Fourier inverse de la partie : $1,3-2,3 \AA$ :

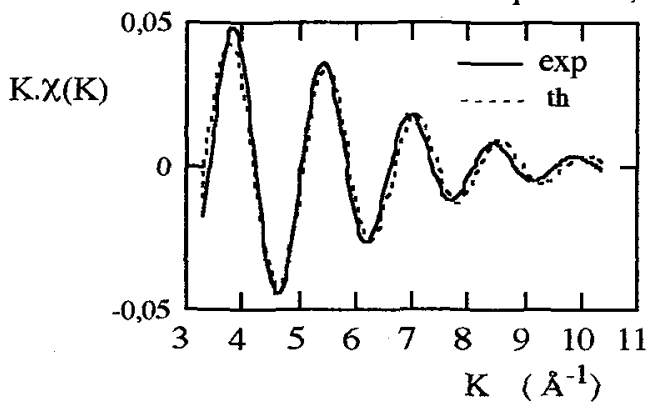

Figure 10: Catalyseur réduit

Fourier inverse $: 1,3-2,3 \AA$

Reduced catalyst

Résultats :

Inverse F T : $1.3-2.3 \AA$

0,5 oxygène $\quad$ à $2,02 \AA$

Simulation de la Fourier inverse de la zone des premiers voisins : $1,3-3,4 \AA$ :

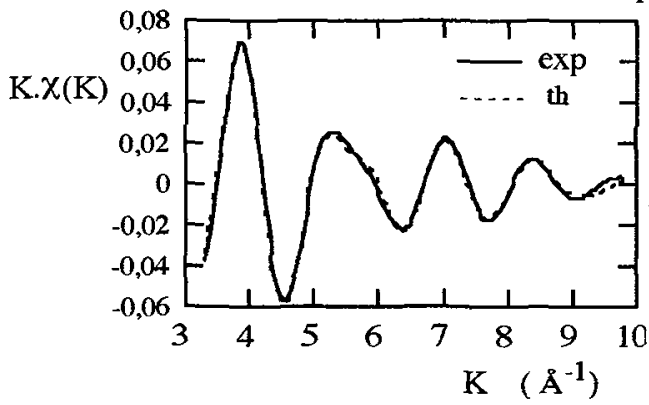

Figure 11 : Catalyseur réduit

Fourier inverse ; $1,3-3,4 \AA$

Reduced catalyst

Inverse F T : $1.3-3.4 \AA$

Résultats à $400^{\circ} \mathrm{C}$
2,0 platine
à $2,79 \AA$,
$\mathrm{DB}=0,08$
1,4 étain
à $2,72 \AA$,
$\mathrm{DB}=0,08$
0,5 oxygène
à $2,01 \AA$,
$\mathrm{DB}=0,015$
0,6 chlore
à $2,32 \AA$,
$\mathrm{DB}=0,04$

\section{DISCUSSION}

L'analyse "EXAFS" permet d'obtenir des informations sur le voisinage du platine pour les différents échantillons. Mais les valeurs proposées pour chaque type de voisin $j: N_{j}$ et $R_{j}$, sont des moyennes par rapport à l'ensemble des voisins du platine.

\subsection{Le catalyseur oxydé}

Sur l'échantillon oxydé, le nombre de voisins oxygène autour du platine est important, ce qui signifie que le platine est en grande partie à l'état d'oxydation $\mathrm{Pt}^{4+}$. Les distances $\mathrm{Pt}$ $\mathrm{O}$ correspondent à la distance $\mathrm{Pt}-\mathrm{O}$ dans $\mathrm{PtO}_{2}: 2,04 \AA$.

La teneur massique en chlore du catalyseur est ajustée à $1 \%$ lors d'un traitement d'oxychloration qui favorise la formation d'oxydes de type $\left[\mathrm{PtO}_{\mathrm{x}} \mathrm{Cl}_{\mathrm{y}}\right.$ ] [2]. L'analyse EXAFS réalisée sur l'échantillon oxydé montre qu'un atome de platine a en moyenne 2,3 atomes de chlore comme premiers voisins. D'après ce résultat, une partie du platine est sous forme d'oxyde du type [ $\left.\mathrm{PtO}_{\mathrm{x}} \mathrm{Cl}_{\mathrm{y}}\right]$. La formation de ces oxydes est importante et d'après Lieske $e t$ al. [3], la redispersion du platine au cours de la régénération n'est observée que si des oxydes du type $\left[\mathrm{PtO}_{\mathrm{x}} \mathrm{Cl}_{\mathrm{y}}\right]$ sont formés.

Sur l'échantillon oxydé, on observe aussi autour du platine des voisins platine et étain. Sous atmosphère oxydante, une partie du platine et de l'étain semble être à l'état métallique. $D^{\prime}$ après Lieske et al. [19], sur un catalyseur $\mathrm{Pt} / \mathrm{Al}_{2} \mathrm{O}_{3}$, l'oxyde de platine $\mathrm{PtO}_{2}$ se décompose en platine métallique sous air si la température est supérieure à $600^{\circ} \mathrm{C}$. La formation de cristallites de platine sur le catalyseur neuf peut être expliquée par la méthode de préparation de ce catalyseur $\mathrm{Pt}-\mathrm{Sn} / \mathrm{Al}_{2} \mathrm{O}_{3}-\mathrm{Cl}$. Les dernières étapes de cette préparation sont une calcination $\overrightarrow{\mathrm{a}} 530^{\circ} \mathrm{C}$ pendant $2 \mathrm{~h}$ et une oxychloration $\mathrm{a} 500^{\circ} \mathrm{C}$ pendant environ $8 \mathrm{~h}$. Ces 
traitements à haute température peuvent expliquer la formation de cristallites de platine métallique de très faible taille (le platine ne voit que 2,4 voisins platine) [20].

D'après Handy et al. [21] et Chojnacki et al. [22], l'alliage PtSn n'est pas formé sous atmosphère oxydante. On peut donc expliquer la présence de voisins étain autour du platine par le fait que les cristallites de platine métallique soient recouvertes par une couche de $\mathrm{SnO}_{2}$. Ces résultats EXAFS montrent que le platine et l'étain se trouvent à proximité l'un de l'autre sur le catalyseur à l'état oxydé.

\subsection{Le catalyseur réduit}

Sur le catalyseur réduit, on observe au voisinage du platine 0,5 oxygène et 0,6 chlore. Ces valeurs sont très faibles et elles sont de l'ordre de la limite d'erreur sur le nombre de voisins. Sur un catalyseur $\mathrm{Pt}-\mathrm{Sn} / \mathrm{Al}_{2} \mathrm{O}_{3}$ réduit, Meitzner et al. [16] observent au voisinage du platine $3,2 \mathrm{Pt}$ et $0,5 \mathrm{O}$. Lorsque le catalyseur est très dispersé, le nombre moyen d'oxygène ou de chlore présent autour du platine peut être d'environ 0,5 . Ces atomes d'oxygène et de chlore sont situés à l'interface entre les cristallites de platine métallique et la surface de l'alumine. Sur le catalyseur $\mathrm{Pt}-\mathrm{Sn} / \mathrm{Al}_{2} \mathrm{O}_{3}$ réduit, les cristallites de platine sont posées à la surface du catalyseur sur une couche d'oxyde d'étain, cet oxyde d'étain étant très fortement lié à l'alumine [5][6]. .

Sur le catalyseur réduit, on observe au voisinage du platine $2 \mathrm{Pt}$ et $1,4 \mathrm{Sn}$. Le nombre global de voisins est très faible ce qui montre que les cristallites métalliques sont très dispersées. Ce résultat est en accord avec ceux obtenus par l'analyse, en microscopie électronique à transmission [23], de cet échantillon réduit.

D'après la distance $\mathrm{Pt}$ - Sn $(2,72 \AA)$, l'alliage formé est du type PtSn. Lors de l'analyse "EXAFS" de catalyseurs réduits $\mathrm{Pt}-\mathrm{Sn} / \mathrm{Al}_{2} \mathrm{O}_{3}$, Meitzner et al. [16] observent moins de 0,5 atome de Sn au voisinage de Pt. Done d'après ces auteurs, aucun alliage est formé. El Abed [24] et El Qebbaj [18] ont analysé par "EXAFS" au seuil LII du platine et au seuil K de l'étain, des échantillons réduits $\mathrm{Pt}-\mathrm{Sn} / \mathrm{Al}_{2} \mathrm{O}_{3}$. Ils obtiennent les distances suivantes:

$$
\begin{array}{lll}
\text { - au seuil LIII du Pt: } & \mathrm{Pt}-\mathrm{Pt}=2,76 \AA \text { et } \mathrm{Pt}-\mathrm{Sn}=2,72 \AA \text {, } \\
\text { - au seuil K de } \mathrm{Sn}: & \mathrm{Sn}-\mathrm{Pt}=2,72 \AA . &
\end{array}
$$

Ces auteurs concluent que l'alliage formé est le Pt-Sn. Nous n'avons pas réalisé d'analyse au seuil de l'étain, mais nos résultats au seuil du platine sont en accord avec les résultats obtenus par El Qebbaj. Si tout le platine est engagé dans un alliage PtSn, il a comme voisin 2 Pt et 6 $\mathrm{Sn}$, le rapport $\mathrm{N}_{\mathrm{P}} / \mathrm{N}_{S n}$ étant de 0,33. Sur le catalyseur réduit, on obtient un rapport $\mathrm{N}_{\mathrm{Pt}} / \mathrm{N}_{\mathrm{Sn}}$ égal à 1,43 . Le pourcentage de platine engagé dans l'alliage PtSn par rapport au platine global est de 23\%:

$$
\frac{\mathrm{N}_{\mathrm{Sn}} \cdot 0,33}{\mathrm{~N}_{\mathrm{Pt}}}=\frac{1,4 \cdot 0,33}{2,0}=0,23
$$

Ce pourcentage d'alliage sur le catalyseur, déterminé à partir des résultats "EXAFS", correspond à celui obtenu par la méthode de titrage avec $\mathrm{H}_{2}$ et $\mathrm{O}_{2}$ [23]. La formation de l'alliage est favorisée par la proximité des atomes de platine et d'étain sur le catalyseur à l'état oxydé [9].

Sur le catalyseur neuf réduit, environ $80 \%$ du platine global est sous forme de petites cristallites de platine et $20 \%$ du platine est engagé dans un alliage PtSn.

La température de réduction $\left(450^{\circ} \mathrm{C}\right)$ est trop faible pour réduire l'étain à l'état métallique si l'étain ne se trouve pas à proximité du platine. Il ne se forme pas de cristallites de $\mathrm{Sn}^{0}$ qui aient ségrégué du platine [6]. Comme sur notre catalyseur le rapport molaire entre le platine et l'étain est de un, il reste donc sur le catalyseur réduit environ $80 \%$ de l'étain à l'état oxydé en forte interaction avec l'alumine ; cette valeur est en accord avec celle déterminée par spectroscopie Mössbauer par Hobson et al. [6]. 


\section{CONCLUSION}

L'analyse EXAFS réalisée in situ du catalyseur $\mathrm{Pt}-\mathrm{Sn} / \mathrm{Al}_{2} \mathrm{O}_{3}-\mathrm{Cl}$, permet de mettre en évidence les points suivants :

- lorsque le catalyseur est régénéré, des oxydes du type $\left[\mathrm{PtO}_{\mathrm{x}} \mathrm{Cl}_{\mathrm{y}}\right]$ sont formés et les oxydes de platine et d'étain sont uniformément répartis et restent en contact,

- lorsque le catalyseur est réduit, la réduction du platine est presque complète, les cristallites métalliques sont très finement dispersées et environ $20 \%$ du platine forme un alliage du type PtSn avec l'étain.

\section{Remerciements.}

Les auteurs remercient M. Guérin (Université de Poitiers) pour les fichiers références, $F$. Maire (LERCSI) et A. Bensaddik (LERCSI) pour leur aide.

\section{Références.}

[1] Kutznetsov V.I., Belyi A.S., Y urchenko E.N., Smolikov M.D., Protasova M.T., Zatolokina E.V., Duplyakin V.K., J. Catal., 99 (1986) 1591.

[2] Lietz G., Lieske H., Spindler H., Hanke W., Volter J., J. Catal., 81 (1983) 17.

[3] Lieske H., Volter J., J. Catal., 90 (1984) 96.

[4] Kappenstein C., Saouabe M., Guerin M., Marecot P., Uszkurat I., Paal Z., Catal. Lett., 31 (1995) 9.

[5] Adkins S.R., Davis B.H., J. Catal., 89 (1984) 371.

[6] Hobson M.C. Jr., Goresh S.L., Khare G.P., J. Catal., 142 (1993) 641.

[7] Bacaud R., Bussière P., Figueras F., J. Catal., 69 (1981) 339.

[8] Dautzenberg F.M., Helle J.N., Biloen P., Sachtler W.M.H., J. Catal., 63 (1980) 119.

[9] Baronetti G.T., De Miguel S.R., Scelza O.A., Castro A.A., Appl. Catal., 24 (1986) 109.

[10] De Miguel S.R., Baronetti G.T., Castro A.A., Scelza O.A., Appl. Catal., 45 (1988) 61.

[11] Sachdev A., Schwank J., Proc. $9^{\text {th }}$ Int. Cong. Catal., Phillips M.J. and Terman M. (Eds.), Calgary, 3 (1988) 1275.

[12] Stencel J.M., Goodman J., Davis B.H., Proc. 9th Int. Cong. Catal., Phillips M.J. and Terman M. (Eds.), Calgary, 3 (1988) 1291.

[13] Davies C.G., Donaldson J.D., J. Chem. Soc., A (1968) 946.

[14] Maire F., Thèse, Université Louis Pasteur, Strasbourg (1994).

[15] Sayers D.E., Stern E.A., Lytle F.W., Phys. Rev. Lett., 27 (1971) 1204.

[16] Meitzner G., Via G.H., Lytle F.W., Fung S.C., Sinfelt J.H., The Journal of Physical Chemistry, 92, ${ }^{\circ} 10(1988) 2925$.

[17] A. Michalowicz, Structure d'adsorption X en chimie: logiciels d'analyse EXAFS pour le Mac., Vol III, Ecole du CNRS, Dexpert H., Michalowicz A.. and Verdaguer M. (Eds.), Garchy (1988).

[18] El Qebbaj S.E., Thèse, Université de Poitiers (1994).

[19] Lieske H., Lietz G., Spindler H., Volter J., J. Catal., 81, (1983) 8.

[20] Caballero A., Villain F., Dexpert H., Lepeltier F., Didillon B., Lynch J., Catal. Lett., 20 (1993) 1 .

[21] Handy B.E., Dumesic J.A., Sherwood R.D., Baker R.T.K., J. Catal., 124, (1990) 160.

[22] Chojnacki T.P., Schmidt L.D., J. Catal., 129, (1991) 473.

[23] Bourges P., Thèse, Université Louis Pasteur (1995).

[24] El Abed A.E., Thèse, Université de Poitiers (1993). 